\title{
Extrapulmonary tuberculosis in the European Union and European Economic Area, 2002 to 2011
}

A Sandgren (andreas.sandgren@ecdc.europa.eu) ${ }^{1}$, V Hollo $^{1}, \mathbf{M}$ J van der Werf ${ }^{1}$

1. Tuberculosis Programme, European Centre for Disease Prevention and Control, Stockholm, Sweden

Citation style for this article:

Sandgren A, Hollo V, van der Werf MJ. Extrapulmonary tuberculosis in the European Union and European Economic Area, 2002 to 2011 . Euro Surveill.

2013;18(12):pii=20431. Available online: http://www.eurosurveillance.org/ViewArticle.aspx?Articleld=20431

Tuberculosis (TB) is decreasing in the European Union/ European Economic Area (EU/EEA), but remains a significant public health problem. Although pulmonary TB accounts for the majority of the cases and is the main transmissible form of the disease, extrapulmonary TB also contributes to the burden of disease and does not receive specific attention in international control strategies. We performed a descriptive analysis to assess the burden and trends of extrapulmonary TB in EU/EEA countries. During 2002-11, 167,652 cases of extrapulmonary TB were reported by the 30 Member States. Extrapulmonary TB accounted for $19.3 \%$ of all notified cases, ranging from $5.8 \%$ to $44.4 \%$ among the Member States. Overall, TB notification rates decreased in 2002-11 due to a decrease in pulmonary TB. Notification rates of extrapulmonary TB remained stable at 3.4 per 100,000 in 2002 and 3.2 per 100,000 in 2011 . Thus the proportion of extrapulmonary TB increased from $16.4 \%$ in 2002 to $22.4 \%$ in 2011. Of all extrapulmonary TB cases reported during $2002-11,37.9 \%$ were foreign-born or citizens of another country, $33.7 \%$ were culture-confirmed, and the overall treatment success was $81.4 \%$. A significant percentage of notified TB cases are extrapulmonary, and in contrast to pulmonary TB, extrapulmonary TB rates are not decreasing.

\section{Introduction}

Tuberculosis (TB) is primarily a disease of the lungs (pulmonary TB), but can affect almost any organ in the body. The term extrapulmonary TB is used to describe the occurrence of TB at sites other than the lung. The most common sites of extrapulmonary TB are lymph nodes, genitourinary tract, pleura, bones and joints, meninges and the central nervous system, peritoneum and other abdominal organs [1-3]. Tuberculosis also exists in a disseminated (miliary) form, with a general bacteraemia spreading the infection throughout the body [4].

Of the 6.2 million cases of TB in the world notified to the World Health Organisation (WHO) in 2011, 5.8 million were new cases, and of the latter, 0.8 million (15\%) cases had extrapulmonary TB [5]. In the WHO Europe region, 253,769 new cases of TB were notified, of which 42,489 (17\%) had extrapulmonary TB [5].

Extrapulmonary TB is rarely addressed in the public health literature. There are however many clinical case reports and case series published, describing patients with different forms of extrapulmonary TB [6-8]. In these publications, extrapulmonary TB is often perceived more as a clinical peculiarity than a public health problem. A reason why extrapulmonary TB is not given high priority on the public health agenda is probably that it does not contribute significantly to the transmission of the disease, very much the same reasoning as used for childhood TB $[9,10]$. Patients with extrapulmonary TB do not receive specific attention in international TB control strategies $[11,12]$. However, extrapulmonary TB contributes significantly to TB-related morbidity and can cause complications, lifelong sequelae and disabilities [1,13-15]. From a public health perspective, there is therefore a need to address this group of patients, as they do contribute to the total burden of disease and they do have a significant impact on available resources of national health systems.

Trend analyses of extrapulmonary TB have been conducted, among others in the Netherlands, Serbia, Spain, the United Kingdom, and the United States (US) [16-21]. An in-depth analysis of extrapulmonary TB in the Member States of the European Union (EU) and European Economic Area (EEA) has not been undertaken. The analysis presented here aims to provide a descriptive overview of the trends in extrapulmonary TB notifications, diagnosis, and treatment outcome during the last 10 years, 2002 to 2011.

\section{Methods}

Data source and collection

We performed a descriptive analysis of surveillance data to assess the burden and trends of extrapulmonary TB in EU/EEA countries between January 2002 and December 2011. Data were extracted from The European Surveillance System (TESSy) for the years 2007 to 2011, and from the former EURO-TB network's historical databases for the years 2002 to 2006, held 
at the European Centre for Disease Prevention and Control (ECDC). Data from 30 EU and EEA countries reporting to the ECDC were analysed. For the purpose of the study, country-specific data for pulmonary and extrapulmonary TB cases were extracted for the years of analysis, for both new and retreatment cases.

\section{Data inclusion and surveillance definitions}

We used the definitions and categories provided in the ECDC/WHO report Tuberculosis surveillance and monitoring in Europe 2012 [22]. Definitions of specific relevance for the analyses in this paper are given here.

All TB cases, confirmed, probable or possible, notified at country level for the year of interest were included in the dataset uploaded to TESSy. Possible cases were considered as those who only met clinical criteria. Probable cases were defined by the additional detection of acid-fast bacilli (AFB) with microscopy or of Mycobacterium tuberculosis in a nucleic acid amplification test or granulomata. Confirmed cases were those with a positive culture for $M$. tuberculosis or with detection of AFB with microscopy and of M. tuberculosis in a nucleic acid amplification test. Cases eligible for treatment, but who never started it, were also included for the purpose of this study, as well as cases diagnosed post mortem.

Site of disease was collected through two variables in TESSy: 'major site of disease' and 'minor site of disease'. For the detailed analysis, we used the variable 'major site of TB'. Pulmonary TB was defined as a case with TB affecting the lung parenchyma, the tracheobronchial tree or the larynx. Extrapulmonary TB was defined as TB with non-pulmonary presentations, and including pleural, intra-thoracic lymphatic, extrathoracic lymphatic, spine, bone/joint other than spine, meninges, central nervous system other than meninges, genitourinary, peritoneal/digestive, disseminated and other TB. Site of disease could also be recorded as unknown. Disseminated TB included TB of more than two organ systems, miliary TB and TB in which M. tuberculosis complex has been isolated from the blood. Cases with concurrent pulmonary and extrapulmonary TB were included in the pulmonary TB category.

\section{FIGURE 1}

Notification rates of pulmonary, extrapulmonary and overall tuberculosis, by year and incidence level, EU/EEA Member States, 2002-11

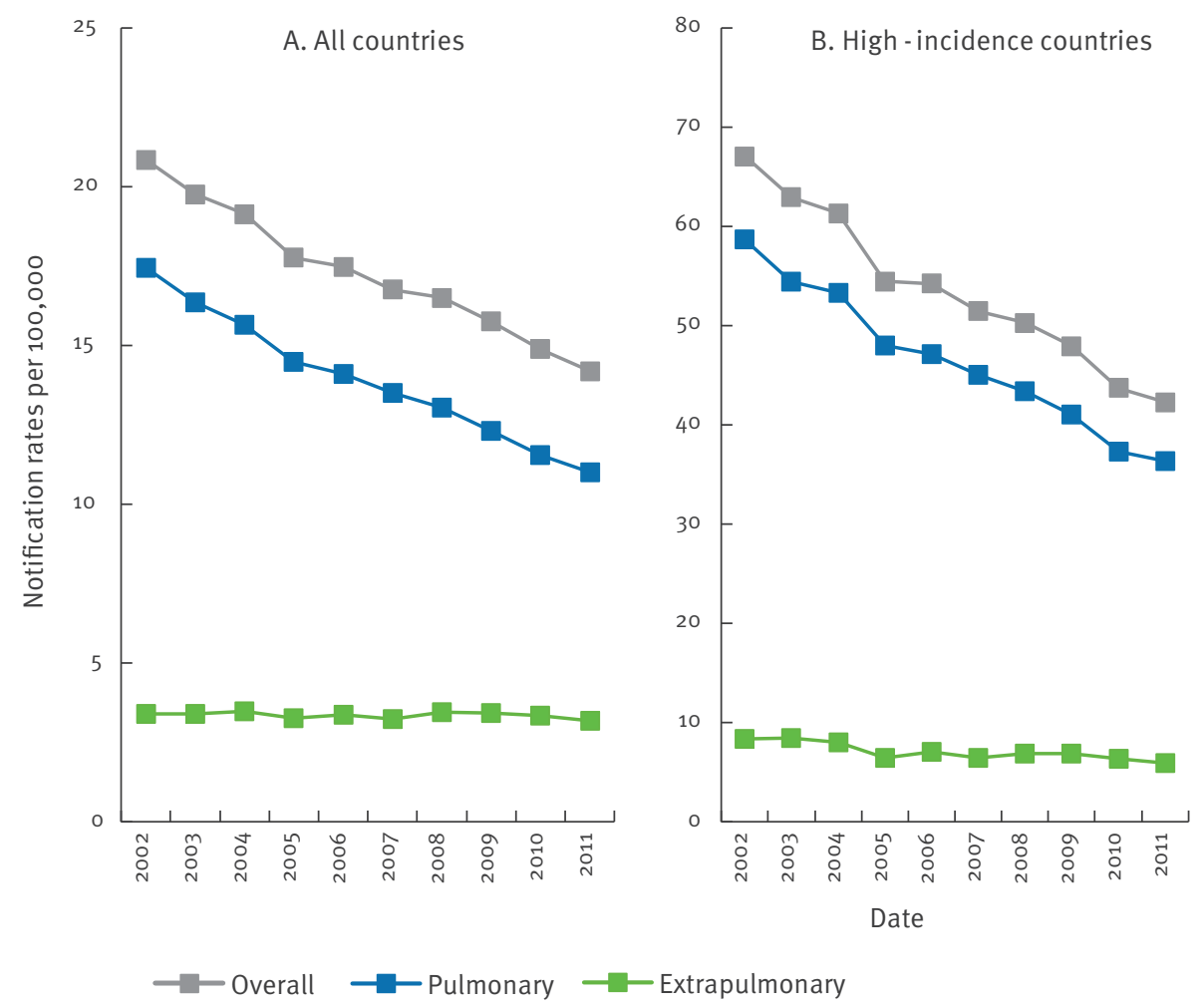

EU/EEA: European Union/European Economic Area.

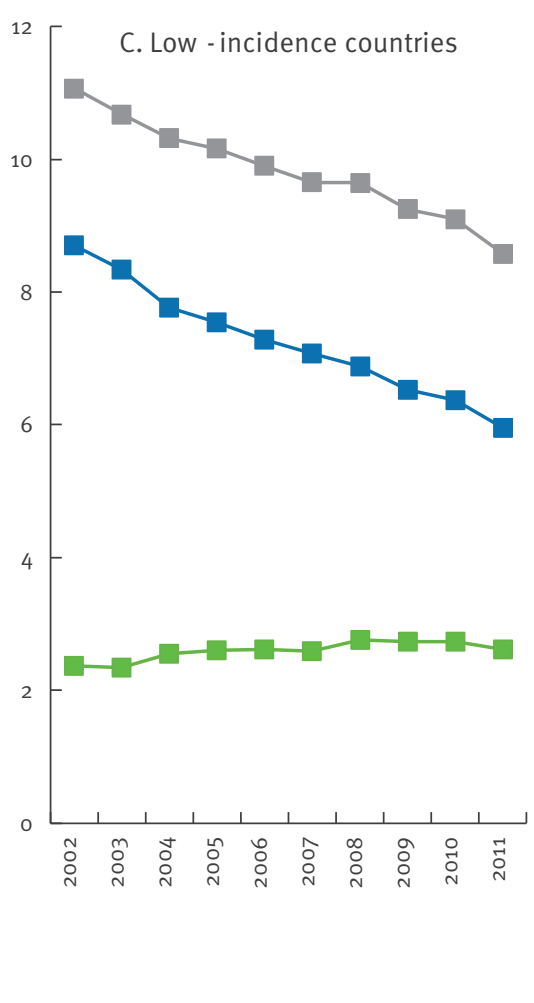




\section{FIGURE 2}

Proportion of extrapulmonary tuberculosis, by year and incidence level, EU/EEA Member States, 2002-11

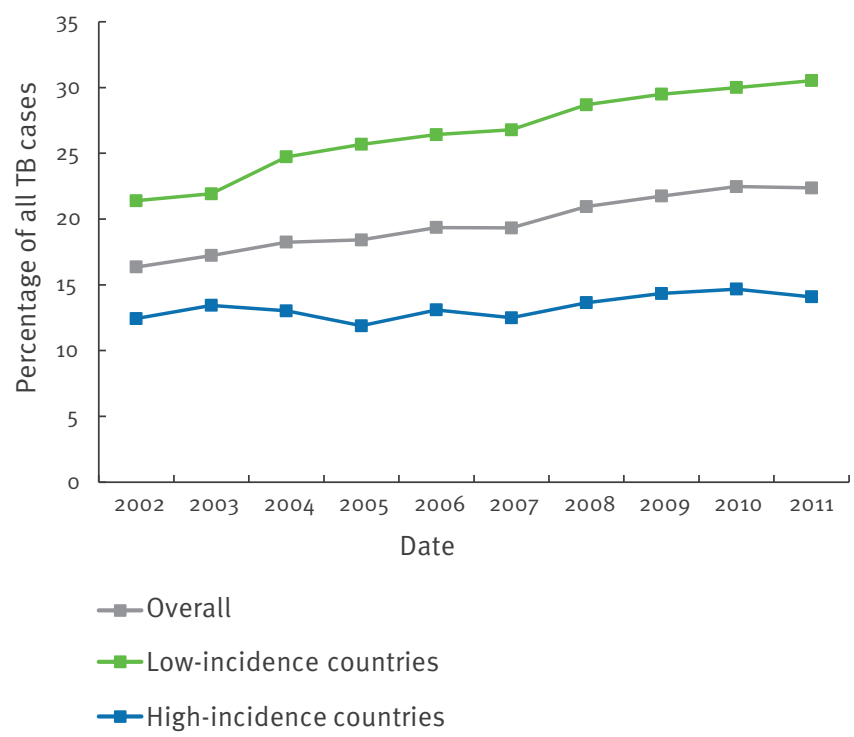

EU/EEA: European Union/European Economic Area; TB: tuberculosis.

The geographical origin of TB cases was classified according to place of birth (born in the country/ foreign-born) or, if unavailable, citizenship (national/ non-national).

\section{Data completeness and quality}

The data uploaded to TESSy went through automated checks for completeness and accuracy. In case-based data collection the probability of case duplications is minimal due to the use of unique record identifiers for reported cases. However, the main responsibility for data quality and correctness lies with the countries that provide the data. Before 2007, 26 of the 29 included EU/EEA Member States were able to report case-based data, for the year 2007 all 30 Member States reported case-based data, and thereafter 29 countries reported case-based data. Comparability of data between countries is compromised by three factors: not all Member States have reported data for the whole period 2002 to 2011, the method of reporting differs by Member State, and some definitions for reporting used by individual Member States are not consistent over time. Therefore, the analyses made in this study have different denominators depending on the variable analysed. We included a specific part in the results section on data completeness, where we specify the number of countries and the respective denominators used for each variable in the analyses.
Analysis

We used StataSE 12 (StataCorp LP, College Station, Texas, US) and Microsoft Excel 2007 for data analyses. Data collected from 2002 to 2011 were collated and tabulated in an aggregated fashion. To be transparent about completeness of data for each variable, we report the unknowns for sex, age groups, origin, previous treatment, human immunodeficiency virus (HIV) infection and TB culture result in the characteristics tables. Percentages have been calculated within the pulmonary TB or extrapulmonary TB strata separately, excluding the unknowns where applicable. Population size was obtained from the EUROSTAT database for 2002 to 2011 (http://epp.eurostat.ec.europa.eu/tgm/ table.do?tab $=$ table $\&$ language $=$ en $\&$ pcode $=$ tps $00001 \&$ tableSelection $=1 \&$ footnotes $=$ yes $\&$ labeling $=$ labels $\& \mathrm{pl}$ ugin=1).

For some analyses, countries were grouped as highand low-incidence TB countries based on the data reported for 2011, using the thresholds previously proposed by the Wolfheze working group [23] and adopted in the EU monitoring framework [24]. Thus, low-incidence countries were defined as those with less than 20 cases per 100,000 population in 2011 ( 23 countries), and high-incidence countries as those with 20 or more cases per 100,000 population in 2011 (seven countries: Bulgaria, Estonia, Latvia, Lithuania, Poland, Portugal and Romania).

Chi-square tests were used to analyse differences in proportions between groups. A p value of $p<0.05$ was considered statistically significant.

\section{Results}

\section{Extrapulmonary tuberculosis \\ notification and trends}

During the period from 2002 to 2011, 868,726 TB cases were reported. For 3,696 of them (0.4\%) the site of infection was not reported, $167,652(19.3 \%)$ had extrapulmonary TB only, 648,225 (74.6\%) had pulmonary TB only, and $49,153(5.7 \%)$ had both and were thus classified as pulmonary TB (total $80.3 \%$ ). The overall proportions of extrapulmonary TB during the study period ranged from $5.8 \%$ to $44.4 \%$ of all TB cases in the different EU/EEA Member States. As the notification rate of pulmonary TB has markedly decreased in most countries of the EU/EEA, the proportion of extrapulmonary TB increased during the period, from $16.4 \%$ of all TB cases in 2002 to $22.4 \%$ in 2011 The notification rates of extrapulmonary TB cases ranged from 0.5 per 100,000 to 13.0 per 100,000 across the EU/EEA Member States for the latest reporting year 2011. During the 10-year period, the overall extrapulmonary TB notification rates remained stable at 3.4 per 100,000 in 2002 and 3.2 per 100,000 in 2011 (Figure $1 \mathrm{~A})$. When stratifying the data by high- and low-incidence countries, the extrapulmonary TB notification rate seemed to be stable in both strata (Figure $1 B$ and Figure ${ }_{1 C}$ ). The proportion of extrapulmonary TB was 


\begin{tabular}{|c|c|c|c|c|c|}
\hline & Pulmonary TB (\%) & Extrapulmonary TB (\%) & Site Unknown (\%) & Total (\%) & $\mathrm{p}$ value \\
\hline Total & $697,378(80.3)$ & $167,652(19.3)$ & $3,696(0.4)$ & $868,726(100)$ & \\
\hline Sex & $\mathrm{N}=642,871$ & $\mathrm{~N}=161,609$ & $\mathrm{~N}=2,744$ & $\mathrm{~N}=807,224$ & $<0.01$ \\
\hline Female & $209,035(32.5)$ & $75,045(46.4)$ & $1,243(45.3)$ & $285,323(35.3)$ & \\
\hline Male & $433,170(67.4)$ & $86,317(53.4)$ & $1,472(53.6)$ & $520,959(64.5)$ & \\
\hline Unknown & $666(0.1)$ & $247(0.2)$ & $29(1.1)$ & $942(0.1)$ & \\
\hline Age groups & $\mathrm{N}=642,871$ & $\mathrm{~N}=161,609$ & $\mathrm{~N}=2,744$ & $\mathrm{~N}=807,224$ & $<0.01$ \\
\hline $0-14$ & $20,017(3.1)$ & $12,433(7.7)$ & $560(20.4)$ & $33,010(4.1)$ & \\
\hline $15-24$ & $68,139(10.6)$ & $21,654(13.4)$ & $232(8.5)$ & $90,025(11.1)$ & \\
\hline $25-44$ & $231,186(35.9)$ & $59,234(36.7)$ & $721(26.3)$ & $291,141(36.1)$ & \\
\hline $45-64$ & $206,594(32.1)$ & $36,585(22.6)$ & $588(21.4)$ & $243,767(30.2)$ & \\
\hline$\geq 65$ & $115,656(18.0)$ & $31,438(19.5)$ & $566(20.6)$ & $147,660(18.3)$ & \\
\hline Unknown & $1,279(0.2)$ & $265(0.2)$ & $77(2.8)$ & $1,621(0.2)$ & \\
\hline Origin & $\mathrm{N}=614,199$ & $\mathrm{~N}=156,957$ & $\mathrm{~N}=2,744$ & $\mathrm{~N}=773,900$ & $<0.01$ \\
\hline Foreign & $108,705(17.7)$ & $59,500(37.9)$ & $850(31.0)$ & $169,055(21.8)$ & \\
\hline Native & $489,721(79.7)$ & $92,048(58.6)$ & $1,198(43.7)$ & $582,967(75 \cdot 3)$ & \\
\hline Unknown & $15,773(2.6)$ & $5,409(3.4)$ & $696(25.4)$ & $21,878(2.8)$ & \\
\hline Previous treatment & $\mathrm{N}=642,871$ & $N=161,609$ & $\mathrm{~N}=2,744$ & $\mathrm{~N}=807,224$ & $<0.01$ \\
\hline No & $501,136(78.0)$ & $136,471(84.8)$ & $1,498(54.6)$ & $639,105(79.2)$ & \\
\hline Yes & $99,509(15.5)$ & $8,197(5.1)$ & $113(4.1)$ & $107,819(13.4)$ & \\
\hline Unknown & $42,226(6.6)$ & $16,941(10.5)$ & $1,133(41.3)$ & $60,300(7.5)$ & \\
\hline HIV reported & $\mathrm{N}=80,963$ & $\mathrm{~N}=32,799$ & $\mathrm{~N}=15$ & $\mathrm{~N}=113,777$ & $<0.05$ \\
\hline HIV tested & $37,936(46.9)$ & 7,199 (21.9) & $1(6.7)$ & $45,136(39.7)$ & \\
\hline HIV-infected b & $1,586(4.2)$ & $468(6.5)$ & $0(0.0)$ & $2,054(4.6)$ & \\
\hline Unknown & $43,027(53.1)$ & $25,600(78.1)$ & $14(93.3)$ & $68,641(60.3)$ & \\
\hline Culture result & $\mathrm{N}=446,449$ & $N=149,749$ & $\mathrm{~N}=3,696$ & $\mathrm{~N}=599,894$ & $<0.01$ \\
\hline Positive & $280,921(62.9)$ & $50,405(33.7)$ & $877(23.7)$ & $332,203(55.4)$ & \\
\hline Negative & $96,718(21.7)$ & $62,873(42.0)$ & $643(17.4)$ & $160,234(26.7)$ & \\
\hline Unknown & $68,810(15.4)$ & $36,471(24.4)$ & 2,176 (58.9) & $107,457(17.9)$ & \\
\hline \multicolumn{6}{|l|}{ DST result } \\
\hline Test performeda & $175,553(62.5)$ & $36,217(71.9)$ & $520(59.3)$ & $212,290(63.9)$ & $<0.01$ \\
\hline MDR-TB & $11,554(6.6)$ & $466(1.3)$ & $15(2.9)$ & $12,035(5.7)$ & \\
\hline Treatment outcome reported & $\mathrm{N}=453,449$ & $\mathrm{~N}=109,297$ & $\mathrm{~N}=1,457$ & $\mathrm{~N}=564,203$ & \\
\hline Treatment success & $333,113(73.5)$ & $88,980(81.4)$ & $769(52.8)$ & $422,862(74.9)$ & $<0.01$ \\
\hline
\end{tabular}

EU/EEA: European Union/European Economic Area; DST: drug susceptibility testing; HIV: human immunodeficiency virus; MDR-TB: multidrugresistant tuberculosis; TB: tuberculosis.

a The denominator for the calculation of percentage of drug susceptibility was the number of culture-positive cases.

${ }^{b}$ The denominator for the calculation of percentage of HIV-infected was the number of HIV-tested cases.

higher (p<0.01) in low-incidence countries $(26.4 \%$ of all TB cases) compared with high-incidence countries (13.2\% of all TB cases).

\section{Characteristics of tuberculosis cases}

Extrapulmonary TB was more frequently notified in women than pulmonary TB: $46.4 \%$ of the extrapulmonary TB cases, compared with $32.5 \%$ of the pulmonary TB cases were female (Table 1). Also the age distribution was different. Extrapulmonary TB was more frequently notified in children than pulmonary TB: $7.7 \%$ of the extrapulmonary TB cases were o to 14 years of age, compared with $3.1 \%$ of the pulmonary TB cases (Table 1). Moreover, extrapulmonary TB was more frequently notified in individuals that were of foreign origin $(37.9 \%)$ compared with pulmonary TB cases (17.7\%). The proportion of extrapulmonary TB of all TB cases in individuals of foreign origin in low-incidence countries increased significantly over the period 2002 to 2011 , from $48.5 \%$ in 2002 to $61.1 \%$ in 2011 (p<0.01, 
Figure 3). For high-incidence countries, the time trend in the proportion of extrapulmonary TB cases of all TB cases in individuals of foreign origin was $4.1 \%$ in 2002 and $3.2 \%$ in 2011 (Figure 3).

HIV test results were available for $21.9 \%$ of the extrapulmonary TB cases compared with $46.9 \%$ of pulmonary TB cases ( $p<0.01)$. Extrapulmonary TB cases who had a HIV test result were more frequently ( $p<0.05)$ HIV-positive compared with pulmonary cases $(6.5 \%$ vs. $4.2 \%$; Table 1).

The TB diagnosis was confirmed by a positive culture in only $33.7 \%$ of the extrapulmonary TB cases. Over the same period, $62.9 \%$ of the pulmonary TB cases had a positive culture ( $p<0.01)$. In $24.4 \%$ of the extrapulmonary TB cases, the culture results were unknown (Table 1). The proportion of culture-positive cases increased slightly in low-incidence countries from $34.5 \%$ in 2002 to $37.5 \%$ in 2011 . For high-incidence countries, the proportion of culture-positive cases was stable at 30.0\% in 2002 and $30.3 \%$ in 2011.

For as many as $71.9 \%$ of the culture-positive extrapulmonary TB cases, drug susceptibility testing was performed. Multidrug-resistant TB (MDR-TB) was identified in $1.3 \%$ of the extrapulmonary TB cases compared with $6.6 \%$ of the pulmonary TB cases ( $p<0.01$; Table 1 ).

Treatment success was achieved in $81.4 \%$ of the extrapulmonary TB cases and $73.5 \%$ of the pulmonary TB cases ( $p<0.01$; Table 1).

Extrapulmonary tuberculosis sites of disease The specific site of extrapulmonary TB was reported for only $108,345(64.6 \%)$ of the 167,652 extrapulmonary TB cases. The most frequently reported forms were pleural TB $(39,749$ cases, $36.7 \%)$ and extrathoracic lymphatic TB $(21,812$ cases, $20.1 \%$ ) (Table 2$)$.

The most frequent forms of TB among 9,735 paediatric cases (o-14 years) were lymphatic intrathoracic TB (47.2\%) and pleural TB (18.5\%). Meningeal TB was present in $5.8 \%$ of the paediatric cases compared with $2.9 \%$ for all the other age groups combined. The highest proportion of paediatric cases were observed in lymphatic intrathoracic TB cases (40.9\%) and meningeal TB cases $(17.8 \%)$. The most frequent forms of TB among 22,778 elderly cases (above 65 years) were pleural TB (29.0\%) and lymphatic extrathoracic TB (21.1\%) (Table 2).

Among 27,667 cases of foreign origin, $35.7 \%$ had extrathoracic lymphatic TB, $16.4 \%$ had pleural TB and $13.6 \%$ had intrathoracic lymphatic TB. In contrast, patients of native origin $(n=78,477)$ more frequently had pleural TB (44.4\%) and less frequently extrathoracic lymphatic TB (14.4\%). The highest proportions of cases of native origin were observed among pleural TB cases $(87.7 \%)$ and genitourinary TB cases (81.2\%) (Table 2).

\section{FIGURE 3}

Extrapulmonary tuberculosis cases by year, origin and incidence level, EU/EEA Member States, 2002-11

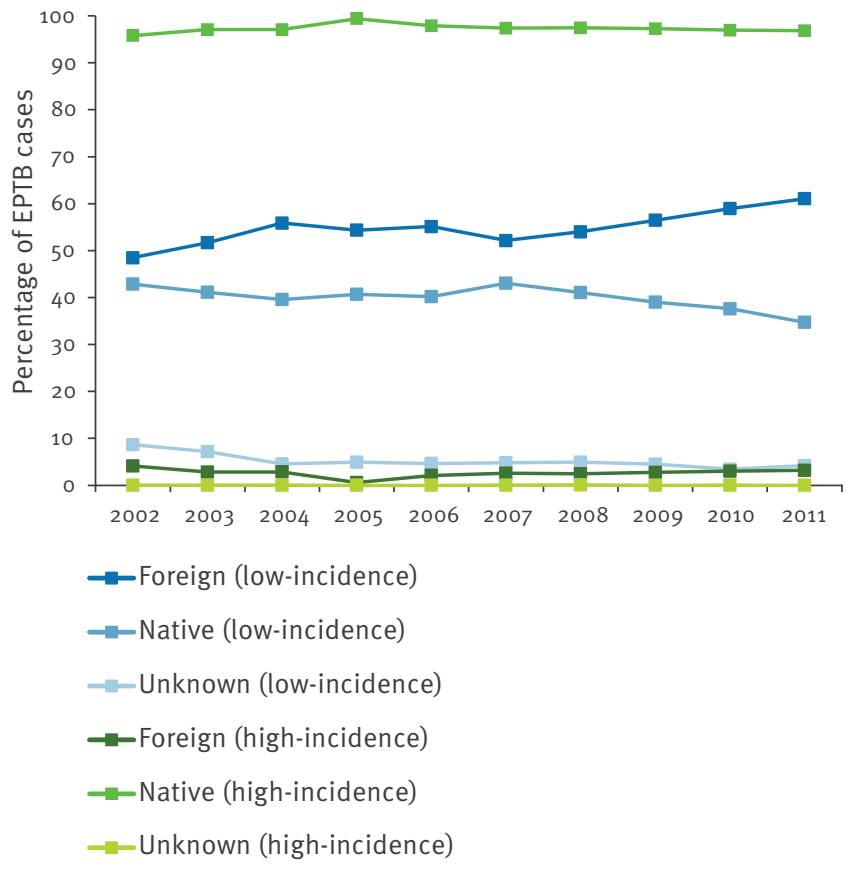

EPTB: extrapulmonary tuberculosis; EU/EEA: European Union/ European Economic Area.

Overall denominator for high-incidence countries: $n=51,356$; overall denominator for low-incidence countries: $n=104,974$.

Low levels of culture confirmation were observed for several of the specific sites. Especially pleural TB cases $(15.1 \%)$ meningeal TB cases $(20.7 \%)$, and spinal TB cases $(21.5 \%)$ were infrequently confirmed by culture. The highest proportions of culture confirmation were observed in genitourinary TB cases (40.8\%) and disseminated TB cases (46.1\%) (Table 2).

Overall treatment success for extrapulmonary TB cases with a known site of disease and with treatment outcome data reported was $83.2 \%$. Only $48.9 \%$ of disseminated TB cases had a successful treatment outcome. Cases with intrathoracic lymphatic TB (82.4\%) and pleural TB $(86.7 \%)$ most frequently had a successful treatment outcome (Table 2).

Tuberculosis notification and data completeness Data completeness varied by country and year for several of the variables for which case-based data were reported (Table 3). There was a marked increase in the number of countries reporting case-based data during the study period for several variables. In particular, data completeness improved greatly for specific site 


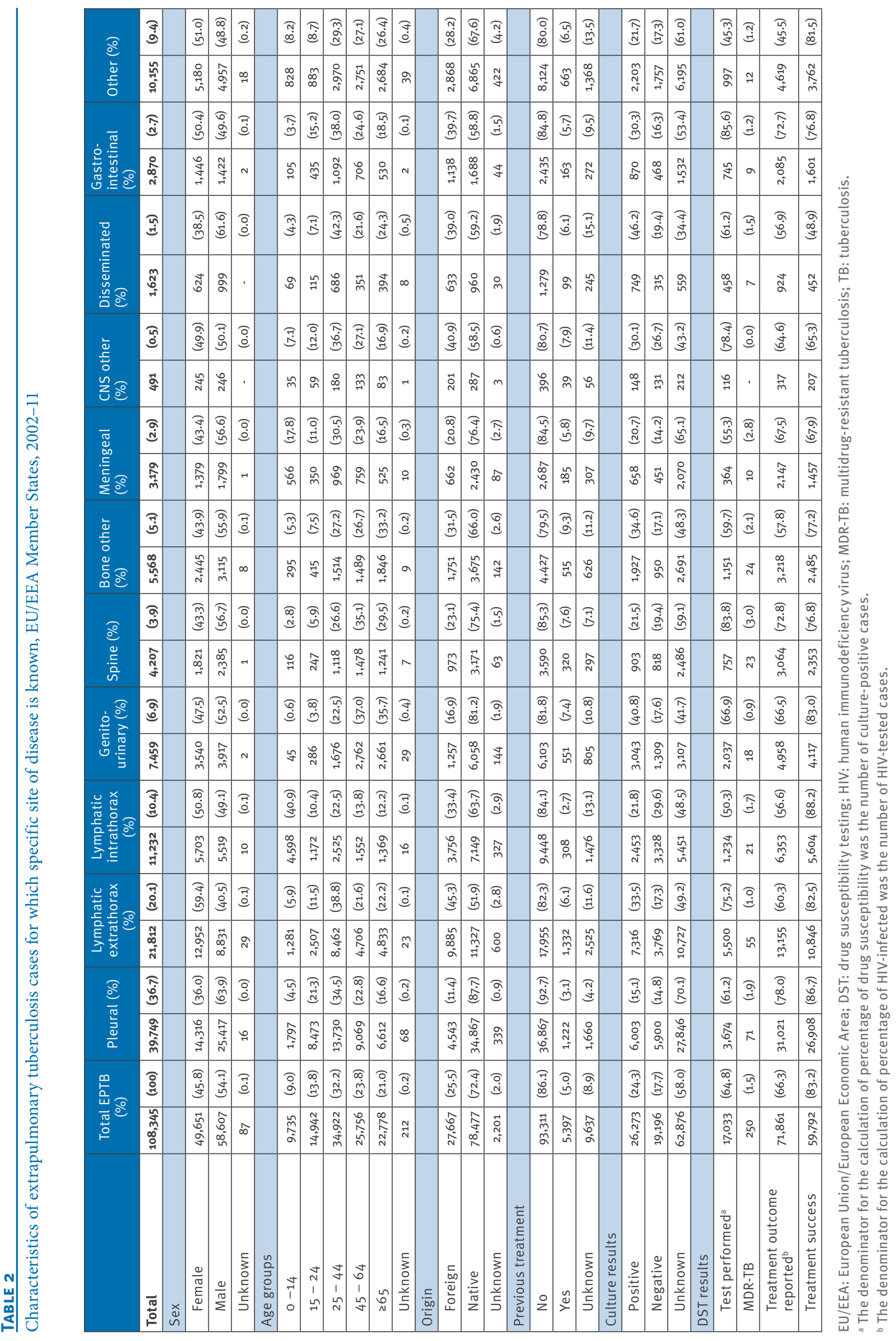




\section{TABLE 3}

Number of EU/EEA Member States reporting data on pulmonary and extrapulmonary tuberculosis and specific site of infection, and total number of cases analysed, 2002-11

\begin{tabular}{|l|c|c|c|c|c|c|c|c|c|c|c|}
\hline Variable & 2002 & 2003 & 2004 & 2005 & 2006 & 2007 & 2008 & 2009 & 2010 & 2011 & Total cases \\
\hline All notified TB cases & 29 & 29 & 29 & 29 & 29 & 30 & 28 & 29 & 29 & 29 & 868,726 \\
\hline Sex & 26 & 27 & 27 & 26 & 27 & 30 & 28 & 29 & 29 & 29 & 816,077 \\
\hline Age & 26 & 27 & 27 & 26 & 27 & 30 & 28 & 29 & 29 & 29 & 816,077 \\
\hline Origin of cases & 23 & 23 & 23 & 25 & 25 & 29 & 28 & 29 & 29 & 29 & 736,594 \\
\hline Previous treatment & 26 & 27 & 27 & 26 & 27 & 30 & 28 & 29 & 29 & 29 & 816,077 \\
\hline HIV status $^{\text {a }}$ & - & - & - & - & - & 29 & 28 & 29 & 29 & 29 & 113,777 \\
\hline Culture confirmation $^{\mid}$ & 25 & 26 & 27 & 25 & 26 & 28 & 26 & 28 & 27 & 27 & 599,894 \\
\hline Drug susceptibility testing & 21 & 22 & 23 & 22 & 23 & 25 & 23 & 25 & 25 & 25 & 212,290 \\
\hline Treatment outcome (12 months)b & 19 & 20 & 20 & 19 & 21 & 21 & 22 & 23 & 24 & - & 564,203 \\
\hline Extrapulmonary TB site specified & 18 & 19 & 20 & 22 & 23 & 26 & 24 & 26 & 26 & 26 & 108,345 \\
\hline Sex & 18 & 19 & 21 & 23 & 23 & 26 & 24 & 26 & 26 & 26 & 108,345 \\
\hline Age & 18 & 19 & 21 & 23 & 23 & 26 & 24 & 26 & 26 & 26 & 108,345 \\
\hline Origin of cases & 17 & 18 & 18 & 21 & 22 & 25 & 24 & 26 & 26 & 26 & 108,345 \\
\hline Previous treatment & 18 & 19 & 21 & 23 & 23 & 26 & 24 & 26 & 26 & 26 & 108,345 \\
\hline HIV status & - & - & - & - & - & 3 & 3 & 5 & 11 & 13 & 4,796 \\
\hline Culture confirmation & 17 & 18 & 19 & 21 & 22 & 25 & 23 & 25 & 25 & 25 & 108,345 \\
\hline Drug susceptibility testing & 14 & 15 & 16 & 17 & 19 & 21 & 20 & 23 & 23 & 23 & 17,033 \\
\hline Treatment outcome (12 months) b & 14 & 15 & 15 & 17 & 19 & 19 & 20 & 22 & 22 & - & 71,861 \\
\hline
\end{tabular}

European Union/European Economic Area; HIV: human immunodeficiency virus; TB: tuberculosis.

Data on HIV status have been collected in TESSy since 2007.

12 month outcome data for a specific treatment cohort are collected in the following calendar year, therefore no data is yet available for the 2011 treatment cohort.

of disease, origin of cases, HIV status, culture confirmation, drug susceptibility testing and treatment outcome.

\section{Discussion}

This is the first descriptive analysis of trends in extrapulmonary TB notifications, diagnosis, and treatment outcome using surveillance data reported by the EU/EEA Member States to ECDC. The overall findings of our study are consistent with other studies performed in geographical regions in and outside of Europe [16$19,21]$. Similar to what has been observed elsewhere, our study shows that the absolute number of notified extrapulmonary TB cases remained stable over the period from 2002 to 2011, but since notification of pulmonary TB cases has decreased, this has led to an increase in the proportion of extrapulmonary TB cases among all TB cases in the EU/EEA.

The partial incompleteness of the data and inconsistencies in reporting over time are limitations when analysing trends and comparing Member States. Overall, the completeness of data has improved over the period of analysis and the efforts to harmonise surveillance data reporting have continuously strengthened the quality and consistency of the data. As data completeness and consistency differ between variables, the pattern of missing data per country and year is very complex. Apart from incompleteness of the data as a result of different data collection and reporting practices across the Member States, there is also a significant risk for under-diagnosis and under-reporting of extrapulmonary cases [25-27]. On the other hand, it can also be argued that there is over-diagnosis of extrapulmonary TB, since only $33.7 \%$ of the cases had their diagnosis confirmed by culture.

Extrapulmonary TB can affect any part of the body, and due to the heterogeneity in clinical manifestations, the diagnosis is especially challenging. Symptoms may be diffuse and mimic other pathologies. Patients present to different specialists who may have little experience in diagnosing tuberculosis and therefore delay reaching the correct diagnosis. This leads to diagnostic delays 
or even missed diagnoses [28]. For these reasons, the analysis of vital registration data and autopsy studies could be helpful when assessing the true burden of extrapulmonary TB.

The notification rates were higher in high-incidence countries of the EU/EEA compared with low-incidence countries, but the proportion of extrapulmonary TB cases was higher in low-incidence countries. One possible cause for this difference is the diagnostic capacity in the different settings. In both settings, the proportion of extrapulmonary TB cases increased as result of the decrease in pulmonary TB over the last decade [29]. Overall, low-incidence countries have a higher proportion of TB cases of foreign origin compared with high-incidence countries [22]. Our study showed that foreign origin is more common among extrapulmonary TB cases. This has also been reported by others $[14,15,17,19]$. We have shown that the proportion of extrapulmonary cases of foreign origin over time remained at a stable low level in high-incidence countries, while it was at a higher level and increasing in low-incidence countries. Thus, a possible factor contributing to the higher proportion of extrapulmonary TB cases in low-incidence countries is the higher overall proportion of individuals of foreign origin. Given the overrepresentation of extrapulmonary TB disease among foreign-born and the increasing presence of foreign-born individuals in several low-incidence countries, it is expected that the proportion of extrapulmonary TB in the EU will increase further. Previous studies that discussed the causes of an increased proportion of extrapulmonary TB acknowledge the association with foreign origin, but also identify other shifts in national population and TB patient demographics [19]. In our study we could not perform further in-depth analyses due to the lack of data on specific risk groups and risk factors.

We observed a higher proportion of TB/HIV co-infection among extrapulmonary cases, with $7.0 \%$ of the tested extrapulmonary TB cases reported to be HIVseropositive compared with $4.2 \%$ of pulmonary TB cases. Due to major lack and inconsistency of data, information on HIV test results stratified by specific site was available for less than $5 \%$ of the HIV-infected patients with extrapulmonary TB. Therefore, we could not confirm the results from a previous study according to which HIV is a risk factor for disseminated TB and concurrent extrapulmonary-pulmonary TB [19].

Our study confirms that MDR-TB is less frequent among extrapulmonary TB cases than among pulmonary TB cases [17]. Given the challenges in diagnosis and obtaining an adequate sample for culture in extrapulmonary $T B$, the treatment regimen is often not based on the drug susceptibility pattern of the infecting strain. Nevertheless, a high treatment success of $81.4 \%$ was achieved.
The proportion of extrapulmonary TB cases that had received previous treatment was very low (5.1\%), as was the proportion of extrapulmonary TB cases with drug resistance. These findings support the hypothesis of Peto et al. [19] that the proportion of extrapulmonary TB cases with previous treatment is very low and therefore the risk of drug resistance is smaller than for pulmonary TB cases.

While the primary aim of further reducing TB transmission by timely diagnosis and adequate treatment of pulmonary TB is paramount for the elimination of TB, due attention should be paid to the group of patients with extrapulmonary TB, who are often neglected in international TB control strategies. In particular, there is a need to raise clinical awareness around the diagnostic challenges posed by extrapulmonary TB. An overview of challenges in diagnosing extrapulmonary TB in the EU is presented in a paper by Solovic et al. in this issue of Eurosurveillance [30]. The proportion of extrapulmonary TB increased during the period from 2002 to 2011, mainly because the notification rate of pulmonary TB decreased. National studies drawing on risk factor data that are not available at EU/EEA level should look further into the specific challenges in each Member State.

\section{Acknowledgements}

The authors would like to thank all the nominated TB surveillance experts of the EU/EEA Member States for providing the surveillance data to TESSy. These are:

Pamela Rendi-Wagner, Maryse Wanlin, Vladimir Milanov, Soteriou Soteroulla, Jiri Wallenfels, Peter Andersen, Piret Viiklepp, Petri Ruutu, Delphine Antoine, Walter Haas, Georgia Spala, Gábor Kovács, Thorsteinn Blondal, Joan O’Donnell, Maria Grazia Pompa, Vija Riekstina, Edita Davidaviciene, Pierre Weicherding, Analita Pace-Asciak, Connie Erkens, Karin Rønning, Maria Korzeniewska-Koseła, Antonio Diniz, Domnica Chiotan, Ivan Solovic, Petra Svetina-Sorli, Elena Rodríguez-Valín, Jerker Jonsson, Ibrahim Abubakar.

Conflict of interest

None declared. 


\section{References}

1. Davies PD, Barnes P, Gordon SB. Clinical Tuberculosis. 4th Edition. London: Hodder Arnold; 2008.

2. Elder NC. Extrapulmonary tuberculosis. A review. Arch Fam Med. 1992;1(1):91-8. http://dx.doi.org/10.1001/archfami.1.1.91. PMid:1341593.

3. Golden MP, Vikram HR. Extrapulmonary tuberculosis: an overview. Am Fam Physician. 2005;72(9):1761-8. PMid:16300038.

4. Sharma SK, Mohan A, Sharma A, Mitra DK. Miliary tuberculosis: new insights into an old disease. Lancet Infect Dis. 2005;5(7):415-30. http://dx.doi.org/10.1016/ S1473-3099(05)70163-8.

5. World Health Organization (WHO). Global tuberculosis report 2012. Geneva: WHO; 2012. Available from: http://apps.who.int/ iris/bitstream/10665/75938/1/9789241564502_eng.pdf

6. Baveja CP, Gumma VN, Jain M, Jha H. Foot ulcer caused by multidrug-resistant Mycobacterium tuberculosis in a diabetic patient. J Med Microbiol. 2010;59(Pt 10):1247-9. http://dx.doi. org/10.1099/jmm.0.019554-0. PMid:20576746.

7. Kriki P, Thodis E, Deftereos S, Panagoutsos S, Theodoridis M, Kantartzi K, et al. A tumor-like manifestation of extrapulmonary tuberculosis in a hemodialysis patient. Clin Nephrol. 2009;71(6):714-8. PMid:19473642.

8. Wiler JL, Shalev R, Filippone L. Case report and review: Potts disease and epididymal tuberculosis presenting as back pain and scrotal mass. Am J Emerg Med. 2010;28(2):261 e3-6.

9. Sandgren A, Cuevas LE, Dara M, Gie RP, Grzemska M, Hawkridge $A$, et al. Childhood tuberculosis: progress requires an advocacy strategy now. Eur Respir J. 2012;40(2):294-7. http://dx.doi.org/10.1183/09031936.00187711. PMid:22337859 PMCid:3409406.

10. Sandgren A, Hollo V, Quinten C, Manissero D. Childhood tuberculosis in the European Union/European Economic Area, 2000 to 2009. Euro Surveill. 2011;16(12):pii=19825. Available from: http://www.eurosurveillance.org/ViewArticle. aspx?Articleld $=19825$

11. European Centre for Disease Prevention and Control (ECDC). Framework action plan to fight tuberculosis in the EU. Stockholm: ECDC; 2008 . Available from: http://ecdc.europa. eu/en/publications/Publications/0803_SPR_TB_Action_plan. pdf

12. Stop TB Partnership, World Health Organisation (WHO). The global plan to stop TB 2011-2015. Geneva: WHO; 2010. Available from: http://whqlibdoc.who.int/ publications/2010/9789241500340_eng.pdf

13. Kim HY, Song KS, Goo JM, Lee IS, Lee KS, Lim TH. Thoracic sequelae and complications of tuberculosis. Radiographics. 2001;21(4):839-60. PMid:11452057.

14. Lenk S, Schroeder J. Genitourinary tuberculosis. Curr Opin Urol. 2001;11(1):93-8. http://dx.doi org/10.1097/00042307-200101000-00014.

15. Malaviya AN, Kotwal PP. Arthritis associated with tuberculosis. Best Pract Res Clin Rheumatol. 2003;17(2):319-43. http:// dx.doi.org/10.1016/S1521-6942(02)00126-2.

16. Garcia-Rodriguez JF, Alvarez-Diaz H, Lorenzo-Garcia MV, Marino-Callejo A, Fernandez-Rial A, Sesma-Sanchez P. Extrapulmonary tuberculosis: epidemiology and risk factors. Enferm Infecc Microbiol Clin. 2011;29(7):502-9. http://dx.doi. org/10.1016/j.eimc.2011.03.005. PMid:21570159.

17. Kruijshaar ME, Abubakar I. Increase in extrapulmonary tuberculosis in England and Wales 1999-2006. Thorax. 2009;64(12):1090-5. http://dx.doi.org/10.1136/ thx.2009.118133. PMid:19850965.

18. Pesut DP, Bulajic MV, Lesic AR. Time trend and clinical pattern of extrapulmonary tuberculosis in Serbia, 19932007. Vojnosanit Pregl. 2012;69(3):227-30. http://dx.doi. org/10.2298/VSP1203227P. PMid:22624407.

19. Peto HM, Pratt RH, Harrington TA, LoBue PA, Armstrong LR. Epidemiology of extrapulmonary tuberculosis in the United States, 1993-2006. Clin Infect Dis. 2009;49(9):1350-7. http:// dx.doi.org/10.1086/605559. PMid:19793000.

20. Rieder HL, Snider DE Jr, Cauthen GM. Extrapulmonary tuberculosis in the United States. Am Rev Respir Dis. 1990;141(2):347-51. PMid:2301852.

21. te Beek LA, van der Werf MJ, Richter C, Borgdorff MW. Extrapulmonary tuberculosis by nationality, The Netherlands, 1993-2001. Emerg Infect Dis. 2006;12(9):1375-82. http://dx.doi. org/10.3201/eid1209.050553. PMid:17073086 PMCid:3294726.

22. European Centre for Disease Prevention and Control (ECDC)/ World Health Organization Regional Office for Europe. Tuberculosis surveillance and monitoring in Europe 2012. Stockholm: ECDC; 2012. Available from: http://ecdc.europa.eu/ en/publications/publications/1203-annual-tb-report.pdf
23. Broekmans JF, Migliori GB, Rieder HL, Lees J, Ruutu P, Loddenkemper R, et al. European framework for tuberculosis control and elimination in countries with a low incidence. Recommendations of the World Health Organization (WHO), International Union Against Tuberculosis and Lung Disease (IUATLD) and Royal Netherlands Tuberculosis Association (KNCV) Working Group. Eur Respir J. 2002;19(4):765-75. http:// dx.doi.org/10.1183/09031936.02.00261402. PMid:11999007.

24. European Centre for Disease Prevention and Control (ECDC). Progressing towards TB elimination. A follow-up to the framework action plan to fight tuberculosis in the EU. Stockholm: ECDC; 2010. Available from: http://www. ecdc.europa.eu/en/publications/Publications/101111_SPR_ Progressing towards TB elimination.pdf

25. Hong SJ, Park YS, An H, Kang SM, Cho EH, Shin SS. Factors leading to under-reporting of tuberculosis in the private sector in Korea. Int J Tuberc Lung Dis. 2012;16(9):1221-7. http://dx.doi. org/10.5588/ijtld.11.0782. PMid:22794136.

26. Migliori GB, Spanevello A, Ballardini L, Neri M, Gambarini C, Moro ML, et al. Validation of the surveillance system for new cases of tuberculosis in a province of northern Italy. Varese Tuberculosis Study Group. Eur Respir J. 1995;8(8):12528. http://dx.doi.org/10.1183/09031936.95.08081252. PMid:7489786.

27. van Hest NA, Smit F, Baars HW, De Vries G, De Haas PE, Westenend PJ, et al. Completeness of notification of tuberculosis in The Netherlands: how reliable is recordlinkage and capture-recapture analysis? Epidemiol Infect. 2007;135(6):1021-9. http://dx.doi.org/10.1017/ So950268806007540. PMid:17156496 PMCid:2870642.

28. Farah MG, Rygh JH, Steen TW, Selmer R, Heldal E, Bjune $G$. Patient and health care system delays in the start of tuberculosis treatment in Norway. BMC Infect Dis. 2006;6:33. http://dx.doi.org/10.1186/1471-2334-6-33. PMid:16504113 PMCid:1435913.

29. Sandgren A, Hollo V, Huitric E, Kodmon C. Epidemiology of tuberculosis in the EU/EEA in 2010: monitoring the progress towards tuberculosis elimination. Euro Surveill. 2012;17(12): pii=20124. Available from: http://www. eurosurveillance.org/ViewArticle.aspx?Articleld=20124. PMid:22490307.

30. Solovic I, Jonsson J, Korzeniewska- Koseła M, Chiotan DI, Pace-Asciak A, Slump E, et al.. Challenges in diagnosing extrapulmonary tuberculosis in the European Union, 2011. Euro Surveill. 2013;18(12):pii=20432. Available online: http://www. eurosurveillance.org/ViewArticle.aspx?Articleld =20432 